\title{
L'enseignement de la philosophie
}

\author{
Claude Collin* \\ Societé Canadienne de Philosophie
}

\begin{abstract}
Cet article porte sur l'enseignement de la philosophie. Il décrit et analyse une remise en question radicale des principaux éléments pédagogiques relatifs à cet enseignement, suite à la création d'un Ministère de l'Éducation au Québec en 1964. Parmi les multiples essais tentés par les professeurs afin de rendre cet enseignement accessible, mesurable et efficace, ce document s'arrête sur un essai de didactique scientifique qui ramène la problématique de l'enseignement philosophique au passage de l'expérience vécue commune à l'expérience philosophique achevée. La méthode utilisée dans l'élaboration de cette didactique s'inspire de la méthode de Raymond Buyse, qui repose sur la notion de fait pédagogique.
\end{abstract}

Mots clés : enseignement, didactique, fait pédagogique, expérience vécue, expérience philosophique.

\section{LA ENSEÑANZA DE LA FILOSOFÍA}

Este artículo trata de la enseñanza de la filosofía. Se describe y se analiza aquí una puesta en cuestión radical de los principales elementos pedagógicos relativos a esta enseñanza, luego de la creación de un Ministerio de la Educación en Quebec en 1964. Entre los múltiples intentos acometidos por los profesores con el fin de volver dicha enseñanza accesible, medible y eficaz, este documento se detiene sobre un ensayo de didáctica científica que conduce la problemática de la enseñanza filosófica del tránsito de la experiencia común vivida a la experiencia filosófica acabada. El método utilizado en la elaboración de esta didáctica se inspira en el método de Raymond Buyse, que se basa en la noción de hecho pedagógico.

Palabras clave : enseñanza, didáctica, hecho pedagógico, experiencia común, experiencia filosófica.

\footnotetext{
* Lic. Théologie, U. Montréal et Lic. Philosophie, U. St. Thomas d'Aquin, Rome. Membre du Comité Directeur de la Société Canadienne de Philosophie
} 


\section{L'ENSEIGNEMENT DE LA PHILOSOPHIE}

Au début des années '60, s'opéra au Québec une révolution culturelle que l'on appela la « révolution tranquille», au cours de laquelle l'enseignement de la philosophie, au niveau collégial, subit une transformation radicale. L'événement déclencheur de cette révolution fut sans doute la création d'un Ministère de l'éducation. Je voudrais, dans les pages qui suivent, marrêter sur ce moment historique précieux, pour le décrire et en marquer la fécondité didactique.

Ce qui fait l'intérêt de cette brève période de transformation c'est qu'elle remettait en question toute la problématique de l'enseignement de la philosophie. La société ellemême passait d'un état stable, paisible, sans trop d'histoire, à un état évolutif doué d'une vitalité nouvelle. Ce fut comme une renaissance pleine de promesses.

J'aborderai donc cette présentation en deux parties : dans un premier temps, je tenterai de décrire l'état de l'enseignement au moment de la création du Ministère ; ensuite, je présenterai un essai de didactique scientifique de la philosophie, qui fut élaborée durant cette période.

\section{Première partie L'état antérieur}

Pour bien cerner l'état précédent de l'enseignement de la philosophie, il faudrait décrire les conditions pédagogiques antérieures, puis analyser brièvement les différentes tentatives de renouveau et, enfin, exposer la problématique de l'enseignement de la philosophie au niveau des cégeps ${ }^{1}$.

\section{Les conditions pédagogiques antérieures}

Comme tous les enseignants de l'époque au Québec (de 1950 à 1960), nous enseignions dans un système que nous connaissions bien, puisque nous l'avions déjà vécu comme étudiants. Ce système s'inspirait de l'humanisme gréco-latin, de la tradition judéochrétienne et de la doctrine sociale de l'Église. Il répondait aux exigences d'une société monolithique, longtemps repliée sur elle-même pour bon nombre de raisons historiques, et qui tardait à entrer dans la modernité.

Dans un tel contexte, le rôle de l'enseignement de la philosophie était clair et tous les agents et responsables de l'éducation le comprenaient. L'enseignement de la logique d'Aristote pouvait répondre parfaitement aux exigences des valeurs intellectuelles recherchées et défendues à l'époque : l'enseignement de la métaphysique et de la théodicée cadrait bien avec les croyances religieuses admises et pratiquées par l'ensemble de la société d'alors; et l'enseignement de la philosophie morale, sociale et politique s'inspirait des valeurs reconnues et partagées par la communauté.

1 Ce sigle désigne : Collège d’Enseignement Général et Professionnel 
On enseignait une philosophie particulière, le thomisme, à l'aide d'un ou deux manuels; il y avait un examen commun qui portait sur les 24 thèses thomistes constituant l'armature de la philosophia perennis.

Dans ce contexte, la philosophie jouissait d'un immense prestige puisqu'elle occupait tout l'espace. Les sciences humaines comportaient l'histoire et la littérature de toutes les époques, en particulier les périodes classiques de la Grèce antique, du Moyen-Âge et de la Renaissance. On s'arrêtait donc aux racines de la culture occidentale. Les sciences mathématiques, la biologie, la physique et la chimie étaient réduites à leurs éléments les plus simples (qui correspondaient d'ailleurs aux exigences universitaires de l'époque). L'enseignement professionnel et l'enseignement technique relevaient d'écoles spécialisées où se donnaient quand même des cours de morale sociale 2 .

Dans ces conditions, il est facile de comprendre quel défi les enseignants de la philosophie durent relever au moment de l'instauration des cégeps. De fait, ce sont toutes les conditions pédagogiques qui changeaient brusquement : apparition d'une population étudiante de 16 et 17 ans comparativement à la précédente de 19-20 ans; programmes fluctuants et indéterminés, méconnaissance du passé culturel de ces nouveaux étudiants, éclatement de la philosophia perennis, enfin, disparition de plus en plus évidente de ce qui constituait les bases de notre culture. De plus, les cours passaient de 7 et 9 heures / semaine à 3 heures / semaine ; et l'ensemble de la matière était réparti en 4 sessions de 45 heures. à la place de tous les traités traditionnels qui s'étalaient de la logique à la morale.

\section{Les essais de renouveau}

Il va sans dire qu'une telle situation pédagogique favorisait une remise en question en profondeur de cet enseignement, car tous les éléments de la pédagogie étaient touchés par cette réforme (le sujet de l'éducation, l'enseignant, l'objectif à atteindre, les moyens à utiliser pour y parvenir et le degré d'apprentissage). Dès lors, on vit naître toutes sortes d'essais, de méthodes, de techniques, de moyens pédagogiques; d'une façon générale, on peut dire que tous ces efforts manifestaient une vitalité rafraîchissante dans le réseau. Ce fut une période exaltante et je crois que dans l'ensemble, les résultats furent plutôt positifs.

On peut signaler plusieurs tendances différentes parmi tous ces efforts :

2.1 Certains s'inspirèrent des plus récents développements de la psychologie, en privilégiant la psychologie de Rogers. "Aucune connaissance n'étant plus certaine, la seule chose que nous puissions enseigner actuellement, affirme Rogers, c'est apprendre à apprendre ».

Il est vrai que cette méthode de Rogers permettait à l'individu de développer son affectivité à l'égard des objets de connaissance, mais elle n'apprenait pas à

2 On comprend pourquoi, avant la création du Ministère de l'Éducation, la recherche en pédagogie de la philosophie n'existait pratiquement pas. Les premiers travaux parurent en 1965 et 1967.

«L'enseignement du Thomisme dans les collèges classiques », Lelievre (1965).

« Thomisme ou Pluralisme», Racette (1967). 
apprendre. Cependant, elle apportait certaines idées-forces comme la nondirectivité, l'auto-évaluation, la dynamique de groupe, une pédagogie de situation.

2.2 Une seconde tendance, sans doute la plus répandue, reposait sur l'expérience des professeurs. D'une part, tout enseignant possède même inconsciemment une connaissance du processus mental propre à la philosophie comme discipline de l'esprit, autrement il ne pourrait même pas enseigner la philosophie. D'autre part, l'ambiguïté de la philosophie elle-même favorisait cette tendance. Nous n'étions plus à l'époque de la philosophie unique de l'ancien système.

En pratique, les enseignants n'avaient pas tellement le choix et faisaient appel à leur expérience.

M. Hubert Grenier, dans son livre sur « La Connaissance Philosophique» ${ }^{3}$ exprime bien cette attitude en rappelant qu'il n'y a pas d'autres moyens d'introduire les autres à la pensée que de penser soi-même.

$\mathrm{Au}$ fond, cette façon d'envisager l'enseignement qu'exprime Hubert Grenier, est bien celle du philosophe.

Nous pouvons donc dire que tout enseignant possède une conception psychopédagogique de l'étudiant, laquelle tend à demeurer à l'état de supposition tant que nous n'avons pas envisagé une méthode de recherche pédagogique capable d'étudier les faits pédagogiques que nous pouvons recueillir dans la classe. En résumé, il s'agit d'un art qui peut être enrichi sans doute par la pédagogie.

2.3 La dernière tendance qu'on pourrait signaler, est celle qui prônait un changement radical du contenu philosophique du programme.

Ainsi, l'enseignement du marxisme connut une certaine popularité. Mais même alors, le problème pédagogique restait entier. On risquait de confondre philosophie et pédagogie. Une philosophie, ou une idéologie ne nous apprend strictement rien sur le processus suivant lequel l'étudiant apprend à philosopher. Une méthode pédagogique porte sur les moyens, (comme par exemple un cours magistral) tandis qu'une méthode philosophique (comme la dialectique, l'analyse) porte sur le processus intellectuel.

On était soucieux de rapprocher le contenu philosophique de la réalité vécue dans notre milieu pour plusieurs raisons : les étudiants n'avaient pas, semble-t-il, la préparation intellectuelle nécessaire, ou du moins, ce n'était plus la même préparation, sans latin ni grec, et de plus, ils appartenaient à une trentaine de concentrations différentes!

Que pouvons-nous conclure de cette description de l'état de l'enseignement de la philosophie au moment de l'instauration du Ministère de l'Éducation?

3 «La Connaissance Philosophique », Grenier, (1967). 
Plusieurs professeurs ont comparé cette période à celle qui prévalut à l'époque de la Révolution française au cours de laquelle on instaura le cours de philosophie ${ }^{4}$. Il s'agissait d'une ère nouvelle où la philosophie cessa d'être considérée comme la servante de la théologie. Il n'y avait plus une philosophie officielle, mais une ouverture sur les connaissances, un appel à l'observation de la réalité vécue à partir de laquelle il fallait réfléchir.

Ce qui ressort de ce brassement d'idées, c'est l'impression qu'une culture nouvelle cherche à se frayer un chemin à travers les éléments d'une culture de moins en moins actuelle. Place aux sciences humaines et même aux technologies nouvelles pour permettre à la jeunesse de se réaliser dans les meilleures conditions.

Il y eut, cela va de soi, une contestation de la philosophie qui n'a pas vraiment cessé depuis le début de la réforme. On reprocha à la philosophie son manque d'unité et son incohérence.

Certains regrettent que le recours aux auteurs anciens ne soit plus aussi exploité qu'autrefois. C'était une source de culture, comme le soutient Allan Bloom ${ }^{5}$. Mais il faut admettre que la culture actuelle se nourrit des découvertes nombreuses et éclairantes des sciences humaines. Par exemple, pour comprendre la géopolitique de l' Amérique du Nord actuelle, rien de mieux qu'un auteur comme Paul Krugman (économiste) ou Emmanuel Todd (sociologue).

Enfin, la réforme permettait à l'enseignement philosophique non seulement de s'ouvrir à la culture du temps présent, mais de repenser la pédagogie de cette matière en profondeur et avec beaucoup plus de précision.

\section{Problématique de l'enseignement de la philosophie}

Dans ces conditions, une didactique scientifique de la philosophie était possible, car nous disposions déjà de tous les éléments permettant l'établissement des faits pédagogiques.

Ces derniers, en réalité, naissent de la pratique effective de l'enseignement de la philosophie. En effet, nous connaissons au moins par ouï-dire, les différentes méthodes qu'utilisent les professeurs (par exemple : exposé magistral, séminaire, travail d'équipe, enseignement audio-visuel). En plus, l'examen attentif des travaux imposés ainsi que celui des questions posées, nous révèle la nature des tâches que doivent accomplir les étudiants. Nous savons aussi qu'il est possible de recueillir des résultats, c'est-à-dire le

4 Dans un article publié dans Le Devoir, (2003/11/15/40526.html.), le philosophe Georges Leroux, propose une nouvelle façon de penser et justifier cet enseignement en tenant compte de l'évolution socioéconomique. Dans l'ancien système l'enseignement de la philosophie s'enracinait dans une culture servante de la théologie; le développement des sciences et des technologies nous invite donc à repenser cet enseignement dans son rapport avec la nouvelle culture.

5 "L'âme désarmée ", Bloom 1987

6 Il suffit, après la lecture de tels auteurs, de passer au second degré de la réflexion, i.e. d'analyser leur prise de position théorique. Ce que le philosophe devrait être en mesure de faire. 
genre de travaux que produisent les étudiants (examens écrits, dissertations, comptes rendus, synthèse, etc.).

L'une des difficultés dans l'élaboration d'une telle recherche réside dans cette notion de faits pédagogiques qu'il est absolument nécessaire de clarifier avant de l'appliquer à la didactique et de lui faire subir le traitement scientifique adéquat. Dans cette recherche, nous nous sommes inspirés de la théorie du Professeur Raymond Buyse, ${ }^{7}$ ainsi que des travaux de L. N. Gage ${ }^{8}$ et de M.Debesse et G. Mialaret ${ }^{9}$.

\section{Précisons tout d'abord ce qu'il faut entendre par « fait pédagogique ».}

Si nous admettons qu'un fait est l'objet d'un jugement reliant deux éléments, à savoir, d'une part, une réalité existante et constatée, et d'autre part, une qualité attribuée à cette réalité, on peut dire que le fait pédagogique implique un jugement reliant deux éléments : un élément constitutif de la réalité scolaire et un autre relevant de l'ordre soit psychopédagogique, sociologique, administratif, ou autre ${ }^{10}$. Ainsi par exemple, les éléments constitutifs du fait pédagogique représentant la réalité scolaire seraient : l'élève, l'enseignant, une méthode décrite, les moyens didactiques utilisés, l'évaluation, le rendement, etc ${ }^{11}$.

D'autre part, les attributions possibles seraient les suivantes : des aspects concernant la psychologie (par exemple : l'élève n'est pas motivé pour un tel travail; ou bien il n'a pas appris l'essentiel de tel concept étudié, etc.) ; des aspects concernant l'administration (i.e. l'enseignant se sert de tel manuel imposé), ou la sociologie, (par exemple : le rendement des écoliers dépend des conditions matérielles et sociales).

Comme nous pouvons le constater, les faits pédagogiques sont multiples et variés. Mais il est évident aussi, que certains faits ne peuvent tout simplement pas faire l'objet d'un examen objectif, parce que l'un ou l'autre des éléments requis pour leur constitution, ne repose que sur la base fragile de l'opinion de celui qui les avait établis.

Il ne suffit pas que l'on soit en possession d'un seul fait ou d'une fréquence quelconque de ce même fait. La science n'est possible que si nous sommes en présence de deux ou plusieurs faits consécutifs dont on mesure le degré de succession nécessaire. Les faits doivent être quantifiables

On pourrait dire que d'un point de vue didactique, le fait pédagogique se définit comme l'expression d'un changement survenu, une modification qui s'est opérée entre un premier état qu'on pourrait appeler l'état initial, et un second état, le point d'arrivée, suite à une activité mentale imposée par l'apprentissage.

7 «L'Expérimentation en Pédagogie », R. Buyse (1935).

8 «Handbook of Research on Teaching ", (1965).

9 «Traité des Sciences Pédagogiques», (1969).

10 Gage (1962).

11 Buyse (1935). 
Il s'en suit qu'à strictement parler, tout fait pédagogique peut comporter plusieurs éléments essentiels, à savoir : le genre d'apprentissage à conférer à l'individu, la tâche précise à accomplir par l'étudiant, et la réaction de l'étudiant face à cette tâche ${ }^{12}$. Ces éléments considérés séparément ne constituent pas, d'un point de vue scientifique, un fait pédagogique, ni même une base suffisante pour l'élaboration d'une didactique. Par exemple, la connaissance des livres utilisés, la détermination des buts spécifiques de la philosophie, l'explication détaillée d'un système pédagogique, certaines méthodes nouvelles et appliquées à l'enseignement de la philosophie (audio-visuel, séminaire, discussion etc.) ne constituent pas, considérées en elles-mêmes, des faits pédagogiques et ne sont pas suffisantes pour l'élaboration d'une didactique. Il faut la conjonction des trois éléments, i.e. la connaissance ou la description de la méthode, la tâche imposée aux sujets et l'expression des résultats.

\section{Seconde partie Une ouverture sur l'avenir}

Après avoir examiné les différentes tendances qui se sont manifestées pour résoudre les problèmes de l'enseignement de la philosophie suite à la création d'un ministère de l'éducation, je voudrais m'arrêter sur la tendance scientifique en raison des possibilités et des aspects nouveaux qu'elle présente. Son approche est inductive (bottom-up) et non déductive (top-down), ce qui lui confère un aspect d'espoir de nouveauté.

Nous aborderons les trois questions suivantes: les travaux préalables, les recherches qu'ils suscitent (les algorithmes et les tâches), et l'élaboration d'une méthode didactique

\section{Les Travaux préalables}

Afin de connaître le changement mental que nous voulons voir se réaliser chez l'étudiant suite à notre enseignement, et être en mesure de l'évaluer, il faudra réaliser un certain nombre de travaux préalables. En effet, il faut d'abord être en mesure de répondre aux questions suivantes:

- comment fonctionnent mentalement les étudiants en rapport avec l'apprentissage de la philosophie?

- qu'attendons-nous des étudiants à qui l'on enseigne la philosophie? (Que faisonsnous lorsque nous enseignons la philosophie?)

Ce sont les deux questions fondamentales. On ne peut répondre à ces questions en se fiant uniquement à son instinct de professeur, à son expérience, ou à ce que la psychologie peut dire à ce sujet. Il s'agit des étudiants que nous avons devant nous. On ne peut se satisfaire d'une opinion.

Alors comment obtenir des réponses valables à ces questions? Ce n'est qu'en étudiant les faits pédagogiques, c'est-à-dire les résultats, que l'on peut y parvenir.

12 Buyse (1935). 
Il en est ainsi, d'ailleurs, de tous les problèmes pédagogiques que l'on rencontre. Nous avons dû faire un grand nombre d'études de faits pédagogiques ${ }^{13}$ pour vraiment connaître les étudiants en rapport avec notre enseignement. La connaissance que nous avons d'eux repose toujours alors sur les faits.

Nous avons effectué plusieurs travaux préalables. Nous aborderons ici les deux principaux qui ont mené à l'élaboration de la méthode didactique. Ils portaient sur l'analyse de la pensée, (qui impliquait sans que nous l'ayons voulu, la critique de la pensée) et l'expérience vécue.

La première étude portait sur l'analyse de la pensée. Nous nous demandions ce que nous cherchons à communiquer à nos étudiants comme connaissance et comme savoirfaire lorsque nous enseignons la philosophie à des débutants. On a alors émis l'hypothèse que nous cherchions à amener nos étudiants à savoir analyser une pensée.

Ainsi nous leur avons demandé d'analyser la pensée suivante : «les cimetières sont remplis de gens qui se croyaient indispensables ».

Nous avons reçu 300 travaux que nous avons analysés pour voir comment réagissaient les étudiants à cette question. Les résultats nous ont éclairés beaucoup ${ }^{14}$.

Ainsi, par exemple, à peine 3\% des étudiants avaient pu identifier les concepts en question. Une bonne majorité ne faisaient que redire en d'autres termes ce que cette pensée leur suggérait à première vue. Plusieurs cherchaient à prouver la justesse de la proposition en faisant appel à leur expérience ou à des connaissance acquises par ailleurs en histoire ou en sciences.

Ce qui nous incita à leur demander, dans un second travail, de raconter une expérience vécue personnelle.

Ce travail fut très éclairant sur une foule d'aspects du comportement intellectuel des étudiants, mais aussi sur bien d'autres aspects utiles pour notre enseignement.

Ainsi, on put dresser une esquisse du profil mental de l'étudiant relativement à l'apprentissage philosophique; on se rend compte que la réflexion de l'étudiant est celle du mode ordinaire de pensée : elle est spontanée, (non structurée) elle se situe au niveau du concret, (non au niveau des idées - il pense par image), elle utilise le langage courant, elle est au niveau du pratique, toujours tournée vers l'action (non au niveau des principes, elle est particulière et close sur elle-même, (non pas ouverte à une remise en question).

Les conséquences de cela sont les suivantes : on croit que toutes les opinions se valent; on pense comme tout le monde, on juge surtout l'image, on est à la remorque de toute prise

13 Dans «L'expérience philosophique », Collin (1978), le lecteur pourra trouver un grand nombre de ces études.

14 Nous avons fait subir ce même test aux étudiants des 4 sessions avec les mêmes résultats. 
de position partagée par l'ensemble, parce qu'on n'a pas de moyen d'en faire la critique, on aime discuter les stratégies d'action.

On put trouver des caractéristiques importantes de l'expérience vécue:

L'expérience vécue dont il est question ici, est l'expérience humaine. On ne doit pas la confondre avec l'expérience scientifique. Elle est extrêmement importante, puisque c'est le chemin de tous les apprentissages; en étudiant l'expérience vécue des étudiants, on peut distinguer les expériences communes, conformes au mode de pensée de tout le monde, et les expériences philosophiques qui sont toute autre chose ${ }^{15}$.

Mais surtout elle a des caractéristiques importantes:

- elle est particulière non seulement au niveau de la description, mais aussi au niveau de l'interprétation et de la conclusion qu'elle implique; ce qui nous éclaire beaucoup sur l'étudiant lui-même; (p.43 à 51 dans « l'expérience philosophique ».) (cf. les notes à la fin de ce texte);

- elle utilise un langage commun;

- elle est interprétative; (p. 56 à 62);

- elle est transformante (p. 62 à 65); ii (réf : notes de la fin de ce texte);

- elle est souvent inconsciente (p. 65 à 71); ${ }^{i i i}$ (réf : notes de la fin de ce texte);

- elle est rapidement généralisatrice.

On put préciser la spécificité de la philosophie ${ }^{16}$; indiquer par quelle partie de la philosophie il serait préférable de commencer la séquence de cours; quels sont les concepts les plus utilisés par les étudiants, quels sont les problèmes philosophiques les plus faciles et les plus fréquents; quelle est la répartition des expériences des étudiants selon l'origine, le sexe, le genre de problème ; nous avons pu dresser une typologie des expériences, enfin une méthode de recherche à la portée des étudiants.

En résumé, nous avons là une mine d’aperçus qui nous éclairent sur les étudiants réels; ils nous aident à déterminer les algorithmes nécessaires à la stimulation de la réflexion. On peut ajouter à tout cela, que nous avons, de la même manière, trouvé une unité de mesure pour évaluer la capacité d'analyse de la pensée; une connaissance des problèmes personnels et sociaux des étudiants.

\section{2- Les recherches}

À partir des résultats de ces études préalables, il devenait possible de chercher les algorithmes capables d'amener les étudiants à réaliser des expériences vécues de type

15 Celles-ci portent sur des idées, sur un énoncé de principe, impliquent une critique, etc.

16 Il s'agit ici de la philosophie qui s'enseigne, (i.e. celle qui est structurée, méthodique, critique) celle qui est comme le chemin de la connaissance de soi et de sa propre pensée. (réf. : "l'Initiation philosophique en 4 leçons, » Collin (1994) p.11 à 14); tout enseignant a en lui-même, consciemment ou non une notion de la philosophie. 
philosophique, à savoir : comment passer de l'expérience commune à l'expérience philosophique proprement dite? ${ }^{17}$

Nous avons été amenés à apporter des précisions très importantes concernant les habiletés intellectuelles qu'il fallait favoriser chez l'étudiant.

Pour que l'expérience vécue prenne un sens, il faut qu'elle devienne philosophique. C'està-dire que l'étudiant sache la rationaliser, la dégager de son caractère particulier, découvrir ce qu'elle nous apprend, l'évaluer. Nous avons pensé que cela était possible si nous divisions en étapes les difficultés à franchir. C'est pourquoi nous avons suggéré de commencer par décrire les faits constitutifs de l'événement, puis de les interpréter et enfin d'identifier la connaissance nouvelle que nous apporte l'expérience. Ce qui nous conduisait à proposer aux étudiants la tâche suivante :

- décrire les faits, les éléments qui constituent l'événement à la base de l'expérience en question,

- interpréter, i.e. dire les raisons d'être (selon vous) de cet événement, les pourquoi, etc.,

- indiquer ce que cela (vous) a appris de nouveau, et l'exprimer sous forme d'énoncé de principe.

Ce questionnement s'est avéré très efficace puisse qu'il permettait à l'étudiant de dépasser le caractère particulier de l'expérience. ${ }^{18}$ Par exemple voici quelques conclusions, sous forme «d'énoncés de principe ».

Une expérience d'exploration :

"L'être humain comble par l'exploration son besoin de connaître. »

Une expérience de musique :

« L'art est un langage qui sert à exprimer une émotion ».

Quand on a réussi à dégager de la description des faits, suivie del'interprétation, un énoncé de principe, on doit se demander ce que vaut cette conclusion qui ressort des faits. On est porté à croire que cette proposition est ce qu'il y a de plus vrai. Mais cela reste à être prouvé. Il faut tout d'abord clarifier cet énoncé pour savoir ce qu'il dit vraiment. Ce qui nous entraine à effectuer une seconde étape, une seconde phase dans cette expérience qui sera éventuellement vraiment philosophique : ce sera la phase de l'analyse de cette pensée.

Les recherches que nous avons effectuées sur cette question, consistaient à découvrir les moyens de clarifier la pensée exprimée dans l'énoncé de principe. Cette clarification s'obtient par l'identification des deux concepts, et du rapport constituant la proposition.

17 Dans «L'Enseignement de la philosophie » Collin (1974), nous exposons comment s'est opéré dans notre recherche la symbiose entre la méthode philosophique et la méthode d'apprentissage. P.35 à 51 Les trois phases de l'apprentissage selon J. Bruner sont : l'information, la transformation et la vérification; lesquelles correspondent aux trois sphères : le vécu, le conçu et la théorie.

18 On trouvera beaucoup d'exemples dans « L'Initiation philosophique en quatre leçons » Collin (1994). 
Pour obtenir l'identification des concepts, il faut souvent opérer une transposition généralisatrice de la proposition. Ce qui constitue la grande difficulté de l'analyse, ${ }^{19}$ mais aussi l'unité de mesure de la capacité d'analyse de la pensée. ${ }^{20}$ Une fois cela réalisé, l'aspect philosophique de l'analyse et le problème philosophique posé par cette position apparaissent. L'aspect philosophique, car on se rend compte que la proposition est un jugement, une prise de position et présente un problème qui est de nature philosophique. C'est pourquoi la démarche demandée aux étudiants pour réaliser cette seconde phase fut la suivante:

1 - identifier les deux concepts de cette prise de position;

2- identifier le rapport mis entre les deux concepts;

3- indiquer le problème philosophique.

Par exemple : supposons que la conclusion était : instruire une nation, c'est la civiliser. Le deux concepts seront : instruction et civilisation. Le rapport entre les deux concepts est ici une relation d'identité. Mais alors, peut-on dire qu'il existe une identité entre l'instruction et la civilisation? Bien sûr que non et c'est là que se situe le problème.

On voit, dès lors, qu'il faudra une autre phase de réflexion pour essayer de solutionner ce problème.

Ainsi, une troisième recherche était donc nécessaire pour voir comment réagiraient les étudiants face à un problème philosophique.

Après étude des résultats nous en sommes venus à proposer graduellement, plusieurs méthodes pouvant être utilisés pour solutionner un problème philosophique. Soit; la dialectique, les quatre questions ultimes proposées par Aristote et la normalité. Sans oublier la nécessité de recourir aux auteurs qui ont déjà abordé ce genre de problème.

\section{3- Élaboration d'une méthode didactique}

Nous nous étions assigné la tâche d'élaborer une méthode didactique tenant compte de l'étudiant réel, des exigences de la philosophie et des facteurs contrôlables permettant une évaluation objective.

Tout cela devait se réaliser dans l'utilisation d'une méthode capable de faire passer l'étudiant d'une expérience commune à une expérience philosophique achevée. En divisant la démarche en étapes qui s'appellent et s'imbriquent l'une dans l'autre et qui tiennent compte de toutes les exigences de la philosophie, nous obtenons une méthode qui est à la portée de l'étudiant et qui respecte toutes les exigences de la pensée philosophique.

Cette méthode permet une évaluation objective tout en assurant l'atteinte du niveau désiré. Ainsi peut-on parler de didactique scientifique de la philosophie.

En fin de compte, la méthode à la quelle nous sommes parvenus offre à chacun la possibilité

19 Cette opération est traitée dans l'initiation philosophique en 4 leçons. Collin (1994), p. 54 et ss.

20 Cette étude paraît dans le site de l'expérience philosophique du cegep du Vieux-Monréal. 
de développer sa propre pensée philosophique, selon son niveau de cours. Toute personne, quel que soit son degré de culture, en franchissant les étapes de cette méthode, atteindra nécessairement le niveau de pensée que l'on reconnaît habituellement à la philosophie, pour la simple raison qu'elle franchira les étapes naturelles d'une réflexion en profondeur.

Nous avons appelé ces étapes naturelles les trois sphères. Chacune a son objet matériel et son objet formel, son procédé propre et son champ culturel.

La sphère du vécu est la "pensée de ce qui arrive ", le premier niveau de la réflexion. Elle est l'élaboration de la pensée personnelle à la recherche du sens. À ce niveau, la recherche philosophique se confond avec tous les éléments de la culture. C'est le premier sens de la philosophie.

Son objet matériel est l'univers en tant qu'il est à la portée de l'homme, c'est-à-dire les faits qui se produisent, les événements, les situations, les phénomènes. Son objet formel est le sens, le rejet du chaos et de l'absurdité; la soif de sens qui répond au désir de se situer, de se familiariser avec l'univers, l'attrait de l'intelligible. Son procédé c'est la description objective, l'interprétation personnelle, l'explication rationnelle, la prise de position qui s'exprime en un énoncé de principe. Son champ culturel : le désir du sens s'épanouit à travers tous les éléments de la culture par lesquels l'homme cherche à répondre à ses besoins fondamentaux : le mythe, la religion, la technologie, les sciences, les institutions sociales, politiques, juridiques, économiques, sportives, etc.

La seconde sphère, i.e. celle du conçu, est la pensée de la parole : c'est le second niveau de la réflexion. Elle est la recherche de la signification et de la pensée libre. Son objet matériel est la parole, le verbe, le langage, le dire, l'expression gestuelle, factuelle ou verbale. L'objet formel est la signification; le discours en tant qu'il renvoie à la pensée (qui est un jugement) dont il est l'expression. C'est le sens au second degré.

Son procédé est l'analyse propositionnelle de la parole par l'identification des concepts, la relation exprimée entre les deux concepts de la proposition, la reformulation claire de la pensée. Cette clarification fait ressentir le besoin d'approfondissement.

L'analyse du discours révèle le problème philosophique, c'est-à-dire le problème du sens de cette prise de position.

Le champ culturel du conçu est l'écriture sous toutes ses formes, littérature, poésie, journalisme; les arts et leurs œuvres, enfin tout ce qui s'inscrit dans un rôle de révélateur de la beauté, du bien ou de la vérité.

La troisième sphère est la sphère de la théorie: elle est la pensée de la pensée. Troisième niveau de la réflexion. Elle est la critique de la pensée; elle recherche l'harmonie entre les sphères du vécu et du conçu. Elle est la philosophie prise au second sens du terme. Elle implique une tendance à aller au fond des choses par une remise en question. Elle est animée par la soif d'amour et de vérité i.e. attirée par la vision ou le désir de la sagesse.

Son objet est la parole sous forme de prise de position, d'énoncé de principe, de jugement. La parole demande à être justifiée, critiquée, évaluée, confirmée. En d'autres termes il s'agit 
de la prise de position en rapport avec un sujet (un thème) qui soulève des interrogations.

L'objet formel est la justification, la confirmation. On est à la recherche de l'harmonie intérieure en justifiant sa propre parole. C'est le développement de la vie intérieure, non seulement va-t-on au fond des choses, mais aussi au fond de soi-même. Le procédé est le suivant : d'abord il s'agit de choisir une méthode (soit celle de la normalité, de la dialectique ou des questions ultimes). Ensuite il y a l'argumentation suivie du recours aux auteurs, enfin la portée de la théorie, i.e. les conséquences, les applications, les remises en question nouvelles.

Le champ culturel est la justification qui s'épanouie dans la mystique et la spiritualité. C'est le supplément d'âme, la dynamique de l'amour et de l'attachement à la vie; c'est une ouverture à toutes les autres formes de la culture. La philosophie est solidaire de l'ensemble de la culture.

\section{Conclusion}

Suite à ces recherches, on peut tirer plusieurs conclusions utiles pour l'enseignement de la philosophie à des débutants.

D'un point de vue didactique, la philosophie n'est pas, elle se construit.

Ce qui fait la valeur de ces prises de position, c'est qu'elles reposent au départ, sur des observations. Bien sûr, il y a beaucoup d'interprétations, mais ce qui est important c'est que le résultat est valable, puisqu'il a été vérifié.

1- L'initiation à la philosophie comporte habituellement deux aspects complémentaires : soit que l'on mette l'accent sur la nécessité de découvrir les connaissances de base indispensables à la compréhension des problèmes philosophiques : ce que l'on pourrait appeler l'aspect informatif de l'apprentissage (la tête bien remplie de Rabelais); soit que l'on préfère insister sur le mode de réflexion propre à la philosophie : ce que l'on pourrait appeler l'aspect formatif de l'apprentissage philosophique (la tête bien faite de Montaigne): en pratique, toute la tradition de cet enseignement présente un dosage entre ces deux formes d'enseignement. La méthode expérientielle fait évidemment partie de l'aspect formatif de l'initiation à la philosophie.

Elle offre à chacun la possibilité de développer sa propre pensée philosophique, selon son niveau de cours. Toute personne, quel que soit son degré de culture, en franchissant les étapes de cette méthode, atteindra nécessairement le niveau de pensée que l'on reconnaît habituellement à la philosophie. Elle franchira les étapes naturelles d'une réflexion en profondeur.

2- Ces tâches soumises aux étudiants correspondent aux dissertations. Mais au lieu d'être un exposé de vérités trouvées, elles constituent une recherche. On fait appel à la raison qui cherche et non à la raison qui a trouvé. 
Il est évident que les cours d'information ont toujours leur place nécessaire dans l'initiation philosophique! Il serait intéressant d'ailleurs d'effectuer des recherches sur cet aspect de la formation philosophique.

3- Cette méthode de recherche à base de faits pédagogique est féconde. Elle apprend à découvrir l'importance de l'expérience vécue humaine, comment la comprendre et pourquoi elle est le devenir même de l'homme.

4- Elle permet d'apprendre l'importance de l'analyse de la pensée pour découvrir les véritables problèmes philosophiques.

5- Enfin, elle permet d'apprendre à situer les textes d'auteurs et à mieux comprendre leur sens et leur raison d'être.

On peut ajouter à ces conclusions, que ces études à base de faits pédagogiques, ont permis de préciser beaucoup de concepts importants comme celui d'expérience; de préciser les opérations mentales propres à la réflexion philosophique comme la recherche du sens, de la signification, la critique philosophique. Elles ont aussi permis de faire une typologie des expériences vécues ${ }^{21}$ et de trouver une unité de mesure de la capacité d'analyse de la pensée.

Les précisions apportées sur l'analyse philosophique et son rapport avec la problématisation ont une certaine importance sur le plan didactique. ${ }^{22}$

Enfin, la méthode proposée aux étudiants réalise une symbiose entre méthode d'apprentissage et méthode philosophique. ${ }^{23}$

En résumé, les exercices effectués à chacune des trois sphères réalisent des objectifs pédagogiques importants. La première sphère apprend à conceptualiser à la façon d'un philosophe; la seconde sphère apprend à analyser une pensée, i.e. un énoncé de principe et la troisième sphère apprend à problématiser. Ce sont sans doute les trois objectifs importants de l'enseignement de la philosophie. Et tout cela peut être mesuré. On peut ainsi avoir des résultats d'évaluation peut être un peu plus objectifs.

Il est important de préciser certains aspects de l'expérience vécue qui apparaissent au cours de ces travaux préalables. C'est pourquoi je voudrais ajouter les notes qui suivent à la fin de ce texte.

21 Méthode de recherche philosophique, Collin (1994).

22 L'initiation philosophique en quatre leçons, Collin (1990).

23 Process of Education, Bruner (1960).

Selon cette théorie, l'apprentissage comporte trois phases : l'acquisition d'une information, la transformation de l'information et la vérification. Ce qui correspond aux trois sphères du vécu, du conçu et de la théorie. 


\section{Sur l'expérience vécue i L'expérience vécue est particulière}

Si l'on demande à l'étudiant d'expliquer les faits constituant le point de départ de l'expérience, iléprouve des difficultés et, bien souvent, ne fait queles redécrire avec plus de détails. Cependant si on lui raconte des faits vécus, il est capable d'en fournir des explications qui peuvent être très variées (d'un étudiant à l'autre) tout en comportant une grande cohérence. L'explication est, en un sens, relative à chacun; elle exprime la compréhension basée sur la perception de l'ensemble des faits ou d'un seul auquel on accorde plus d'importance. Il arrive souvent que l'étudiant isole un fait qui le frappe davantage et en fait le centre de son explication. L'explication demeure toujours personnelle et visiblement inspirée par des connaissances acquises en d'autres domaines. On peut donc dire qu'elle demeure en un certain sens particulière, comme étant vécue et comprise par un sujet particulier.

Il en est de même de la conclusion. Si on demande à l'étudiant ce que lui révèlent ces faits, il tire une conclusion rapide, qui le plus souvent demeure particulière, en ce sens qu'elle porte sur un concret pratique, immédiat. Sa conclusion demeure dans l'ordre stratégique.

\section{ii L'expérience vécue est transformante}

Le caractère transformant de l'expérience vécue est le cœur même de la réflexion philosophique. Il se présente comme l'envers de l'interprétation, comme son étoffe intérieure.

L'originalité de l'expérience se situe d'abord au niveau mental et s'exprime dans l'attitude nouvelle de la personne, qui ne s'explique elle-même que par sa conception nouvelle de la réalité.

Cette caractéristique certainement fondamentale de l'expérience peut nous éclairer sur les moyens d'identifier l'expérience et d'établir les conditions d'une évolution vers un degré supérieur d'expérience. Ce qui peut indiquer en pratique avec certitude, de quelle expérience il s'agit, quel concept central spécifie la totalité organique que constitue l'expérience, c'est l'attitude nouvelle de la personne, son comportement réel suite à cette expérience.

Ce qui signifie aussi que la structure des événements dépend en partie de la personne à qui surviennent ces faits : il en va de même de la valeur respective attribuée à tous les éléments organiques du fait global.

\section{L'expérience vécue a un caractère concret avec une logique du concret}

Ce qui signifie encore que le plus important aspect de l'expérience vécue est son caractère concret, avec sa logique du concret. Cette logique du fait nous marque parce qu'elle est particulière comme le fait lui-même; elle agit sur nous en tant même que particulière et concrète. De plus, c'est la raison pour laquelle toute expérience vécue est sujette à l'erreur, car il n'existe jamais de faits totalement identiques. L'identité n'est totale qu'au niveau de l'essentiel. 


\section{iii L'inconscient de l'expérience vécue.}

Le fait que, dans la plupart des cas, l'étudiant ne fasse que raconter les faits de l'expérience vécue et qu'il ne puisse déterminer le concept central de son expérience qu'en faisant appel à une recherche intellectuelle nous montre bien que le vécu n'est pas toujours conçu explicitement ; mais le fait qu'effectivement son attitude soit changée en rapport avec un conçu qui peut être exprimé explicitement nous porte à croire qu'il existe un conçu implicite, que l'on pourrait assimiler à un conçu pré réfléchi, inconscient, c'est-àdire un concept qui n'est pas nommé, verbalisé, mais dont les déterminations sont présentes activement dans la structure concrète du vécu. Il vient à la conscience par la recherche de l'intelligibilité de la structure du nouveau réel vécu.

Mon comportement me révèle ce que je suis en me révélant ce que je pense inconsciemment. Il y a toujours correspondance entre l'être personnel et la pensée, au sens très large de disposition, mentalité, etc. Quand la pensée consciente n'assume pas intellectuellement la pensée inconsciente, il y a brisure ressentie dans mon corps, avec des effets dans la structure expérientielle et opératoire.

\section{Bibliographie}

Bruner, J. The Process of Education, Vintage Book, New-York, 1960.

Buyse, R. Expérimentation en Pédagogie, Lamertin, Bruxelles, 1935.

Collin, C \& Z. Osana, L'Enseignement de la philosophie, Fides, 1974.

Collin, C. L'Expérience philosophique, Bellarmin, 1978.

Collin, C. Méthode de recherche philosophique, Griffon d'argile, 1990.

Collin, C. L'initiation philosophique en quatre leçons, Griffon d'argile, 1994.

Debesse, M. et Mialaret, G. Traité des Sciences Pédagogiques, Tome I, PUF, Paris, 1969.

Fourastier, J. Faillite de l'Université?, Gallimard, 1972.

Gage, N. L. Handbook of Research on Teaching, McNally \& Company, Chicago, 1962.

Mager, R.F. Comment définir des objectifs pédagogiques? Gauthiers-Villars, Paris, 1971.

Planchard, E., La pédagogie scolaire contemporaine, Éditions Nauwelhaerts, Louvain, 1968.

Recibido: Junio 11 de 2009 - Aprobado: Agosto 10 de 2009 